\title{
Serological screening for Celiac Disease in 382 pre-schoolers with Autism Spectrum Disorder
}

\author{
Sara Calderoni ${ }^{1,2^{*}}$, Elisa Santocchi ${ }^{1}$, Teresa Del Bianco ${ }^{3}$, Elena Brunori ${ }^{1}$, Laura Caponi ${ }^{4}$, Aldo Paolicchi ${ }^{4}$, \\ Francesca Fulceri ${ }^{1}$, Margherita Prosperi ${ }^{1}$, Antonio Narzisi ${ }^{1}, 2$, Angela Cosenza ${ }^{1}$, Raffaella Tancredi ${ }^{1}$ \\ and Filippo Muratori ${ }^{1,5}$
}

\begin{abstract}
Background: Recent investigations suggest a possible common genetic background between Autism Spectrum Disorders (ASD) and Celiac Disease (CD). However, studies regarding this association are scarce and often limited by the small sample sizes and/or large heterogeneity among ASD groups in terms of demographic and clinical features. The present study aims to investigate the overall CD prevalence (biopsy proven-CD patients plus screening detected tTG and EMA positive cases) in a large population of pre-schoolers with ASD referred to a tertiary care University Hospital.
\end{abstract}

Methods: We retrospectively collected data about 382 children (mean age: $46.97 \pm 13.55$ months; age-range: 18-72 months) consecutively diagnosed as ASD (according to the Diagnostic and Statistical Manual of Mental Disorders 4th edition criteria) over the period 2010-2013, and who performed a serological CD screening.

Results: The overall CD prevalence was $2.62 \%$, which is statistically significant higher to that reported in the Italian paediatric population $(p=0.0246)$. Half of these children had no symptoms or risk factors related to CD when they performed the serological screening.

Conclusions: If replicated, these data suggest the importance of regular screening for CD in young patients with ASD, and are of relevance for clinical and public health.

Keywords: Celiac Disease, Autism Spectrum Disorders, Pre-schoolers, Screening, Autoimmune disease, Gastrointestinal symptoms, Epidemiology, Young children

\section{Background}

Autism Spectrum Disorders (ASD) are neurodevelopmental conditions characterized by impairment in socio-communicative abilities as well as restricted and stereotyped behaviours [1]. Besides the aforementioned core symptoms, patients with ASD frequently show a wide range of associated clinical manifestations, including other psychiatric [2], and medical comorbidities [3]. Among these latter, gastrointestinal (GI) dysfunctions are reported by parents of ASD children with a rate of

\footnotetext{
* Correspondence: sara.calderoni@fsm.unipi.it

${ }^{1}$ IRCCS Stella Maris Foundation, Viale del Tirreno 331, Pisa 56018, Calambrone, Italy

${ }^{2}$ Stella Maris Mediterraneo Foundation, Chiaromonte, (PZ), Italy

Full list of author information is available at the end of the article
}

20-85\% depending both on definition of GI symptoms and demographic/clinical characteristics of ASD samples $[4,5]$. Simultaneously, recent evidences suggest a role of immune dysfunction in ASD pathogenesis [6], sustained also by increased rates of autoimmune disorders in the families of subjects with ASD [7-9]. In this framework, a possible association between ASD and Celiac Disease (CD) has been suggested. CD is defined as "a chronic small intestinal immune-mediated enteropathy precipitated by exposure to dietary gluten in genetically predisposed individuals" [10]. The prevalence of CD in Western population is close to $1 \%$ [11]; in particular in Italy it was estimated to be $1.1 \%$ among children aged $0-16$ years [12], and $1.2 \%$ among school children, on the basis of a salivary radio-immunological screening [13]. 
Clinical manifestations in childhood are not limited to classical GI symptoms (diarrhoea, abdominal pain, bloating, flatulence, weight loss, anorexia, constipation), but include other common or atypical manifestations potentially affecting any organ or body system. Moreover, two asymptomatic forms are described: Silent CD characterized by positive serology and histology, and Potential CD with positive serology and compatible Human Leukocyte Antigen (HLA) alleles but negative histology [14, 15]. Several complications are associated with untreated $\mathrm{CD}$, and an increased risk of overall mortality has been observed [16].

The association between ASD and CD is still a matter of debate. Some studies reported no evidence for the existence of a ASD-CD link [17-19], whereas others suggested a possible connection [20], or a higher prevalence of CD in ASD children than in general pediatric population [21]. Ludvigsson and colleagues [22] found that ASD was associated with an increased risk of positive CD serology. It is worth noting that, according to one of the "gut-to-brainconnection" theories in ASD, gluten (and casein) can trigger inflammation in the gut leading to autoimmune illness or cross-reactivity with other potential central nervous system antigens $[23,24]$. Therefore, the supposed relationship between gluten and ASD has paved the way for indiscriminate therapeutic approaches such as gluten (and/or casein) free diet, bereft of scientifically validated benefits in absence of a CD diagnosis, as assessed by two recent reviews $[25,26]$. A further reason for the scientific community to better clarify the possible links of ASD and CD.

The aim of this study was to detect the prevalence of $\mathrm{CD}$ in a large series of carefully diagnosed ASD preschool children referred to a tertiary care University Hospital.

\section{Methods}

\section{Participants}

We retrospectively reviewed data of inpatient and day-patient preschool children who had received a first clinical evaluation with a diagnosis of Autistic Disorder, Asperger's Disorder or Pervasive Developmental Disorder-Not Otherwise Specified according to the DSM-IV-TR criteria [27] at the IRCCS Stella Maris Foundation (Pisa, Italy) between January 2010 and December 2013. The sample was composed of 382 children, 304 males and 78 females with an average age of 46.97 months (standard deviation: 13.55 months; range: $18-72$ months). ASD diagnosis was performed by a multidisciplinary team (a senior child psychiatrist, an experienced clinically trained research child psychologist, an educational therapist, and a speech-language pathologist) during 5-7 days of extensive evaluation, and confirmed by the Autism Diagnostic Observation Schedule-Generic (ADOS-G) [28] in the large majority of subjects. Exclusion criteria were: (a) neurological syndromes or focal neurological signs; (b) significant sensory impairment (e.g., blindness, deafness); (c) potential secondary causes of ASD revealed by high-resolution karyotyping, DNA analysis of Fragile-X, or screening tests for inborn errors of metabolism.

\section{Celiac disease screening and diagnosis}

During the first evaluation in the ASD Operative Unit of our Institute, all children usually undergo a serological screening for $\mathrm{CD}$ as part of the routine assessment through the determination of the titres of Anti-Gliadin (AGA) immunoglobuline (Ig)A and IgG, Anti-Transglutaminase (anti-tTG) IgA, and Anti-Endomysium (EMA) IgA antibodies. The first two antibodies are quantified, respectively, with fully automated EliA ${ }^{\mathrm{Tm}}$ Gliadin and EliA ${ }^{\mathrm{Tm}}$ Celikey $^{\circ}$ on ImmunoCAP 250 (Phadia), and measured with fluorescent enzyme immunoassay (FEIA). The upper limit of their physiological range is $10 \mathrm{U} / \mathrm{ml}$ values; between 7 and $10 \mathrm{U} /$ $\mathrm{ml}$ are considered "borderline"; values under $7 \mathrm{U} / \mathrm{ml}$ are considered negative. EMA IgA is measured with indirect immunofluorescence on Euroimmun slides, and it is subject to significant variability of interpretation, but its specificity is very high (98-100\%) [15]. Possible EMA results in our sample were: "+": positive result; "-": negative result; "+/-": doubt/borderline result. We retrieved the results of the $\mathrm{CD}$ screenings from the digital database of the Clinical Pathology Laboratory of St Chiara Hospital (Pisa, Italy). According to the European Society for Pediatric Gastroenterology, Hepatology, and Nutrition (ESPGHAN) new guidelines for the CD diagnosis [15, 29], the serological diagnosis of $\mathrm{CD}$ is based on the detection of class IgA anti-tTG and EMA antibodies; in patients with IgA deficiency, IgG anti tTG is used. Specifically, the sensitivities for both tTG and EMA antibodies ranged from 70 to $100 \%$ [30]. In a large, prospective, biopsy-confirmed study on adult patients without a previous known diagnosis of $\mathrm{CD}$, the negative predictive value for IgA tTG was 99.6\%, and it increased to $99.7 \%$ when a two-step approach was adopted, using tTG first and EMA then [31]. The specificity for tTG ranged from 91 to $100 \%$, whereas EMA specificity is even higher (98-100\%), so much that this test is considered specific for a diagnosis of $\mathrm{CD}$ [30]. A positivity of both anti-tTG and EMA antibodies has a sensitivity and positive predictive value for CD close to $100 \%$ [30,32]. A confirmation of the $\mathrm{CD}$ diagnosis is recommended to all individuals positive for anti-tTG and/or EMA antibodies. Therefore, we selected from the sample all subjects with positive or borderline values of anti-tTG and/or EMA antibodies at the serological screening and then checked in the patient's medical records or through call interviews whether the $\mathrm{CD}$ diagnosis had been subsequently confirmed by paediatric gastroenterologists. We did not took into account the screening levels of AGA IgA and IgG antibodies for the purposes of this study since they have been recently considered as the less specific markers of 
inflammation due to dietary gluten, their increase may be explained by an array of diseases and its significance is still under debate $[15,29]$.

Since we aimed to compare the overall prevalence of CD in our sample to that found in the general population, we used the data from the survey of Mustalahti et al. [12], which investigated the $\mathrm{CD}$ prevalence in four European countries -Italy included- by analyzing the sera from 29,212 children and adults. The Italian sample of children was composed of 2649 individuals with an age-range from 0 to 19 years (the means and standard deviations values were not reported). The age range of our sample was quite different from that of this European survey [12] (1.5-6 years versus $0-19$ years). Therefore, since the prevalence of $C D$ tends to increase by age [33], an underestimation of $\mathrm{CD}$ prevalence in our ASD population could not be excluded when it is compared to the prevalence of the Italian children included in the European survey, i.e. 1.1\% (95\% CI: $0.7-1.5)$, derived from "previously diagnosed biopsy-proven $\mathrm{CD}$ patients plus screening detected individuals with both anti-tTG and EMA positivity" [12].

\section{Statistical analysis}

Statistical calculations were performed with $\mathrm{SPSS}^{\circ}$ version 19.0. We performed the Chi-square test to compare the prevalence of $\mathrm{CD}$ between our sample and the Italian pediatric population [12]. A $p$-value $<0.05$ was considered significant.

\section{Results}

Among 382 pre-schoolers with ASD, the retrospective review of their medical records identified ten patients with $\mathrm{CD}$ or with positive $\mathrm{CD}$ serology (Table 1 ). In particular, nine subjects resulted serologically positive for anti-tTG IgA and/or EMA antibodies during their first neuropsychiatric evaluation. In seven of these children (patients 1-7), the diagnosis of $\mathrm{CD}$ was confirmed by paediatric gastroenterologists: (a) through multiple duodenal biopsies (subjects 1-5); (b) on the basis of very high anti-tTG antibody levels and HLA positivity (patient 6); (c) on the presence of Duhring-Brocq dermatitis herpetiformis, a chronic bullous disease specifically correlated with sensitivity to gluten enteropathy and $\mathrm{CD}$ (patient 7) [34]. All these patients were on a gluten-free diet (GFD). As far as patient 8 is concerned, we were not able to obtain any further information since his parents were no more contactable after the serological screening. The patient 9 did not perform further evaluations even in the presence of repeated positivity of CD serology due to parents' refusal. The patient 10 was already diagnosed as celiac and was on a GFD at the time of the first clinical evaluation in our hospital. She had performed CD serological screening at the age of 32 months since she showed growth failure and dental enamel defects. She resulted serologically positive for anti-tTG IgA and EMA antibodies, and eventually CD diagnosis was confirmed by multiple duodenal biopsies and HLA positivity.

All in all, patients with $C D$ or with positive $C D$ serology were ten, six males and four females, with mean age of 41 months (SD: 15.2; range: 20-67). Therefore, the prevalence of " $\mathrm{CD}$ patients plus screening detected individuals with both anti-tTG and EMA positivity" was $2.62 \%$ (10/382 subjects).

The Chi square test (see Table 2 ) indicated a statistically significant difference ( $p$ value $=0.0131)$ in the prevalence of CD cases identified in our sample $(2.62 \% ; 95 \% \mathrm{CI}$ : $1.0-4.2)$ as compared to the sample obtained from the Italian paediatric population (1.1\%; 95\% CI: $0.7-1.5)$ [12].

Of the ten subjects with $\mathrm{CD}$ (or positive $\mathrm{CD}$ serology), four patients (40\%: the patients No 1, 5, 7, and 10) showed one or more symptoms suggestive of a typical form of CD. Patient No 3 was asymptomatic, but belonged to a high risk group given a positive familiarity

Table 1 Serology, clinical data and diagnosis confirmation of the ten ASD subjects with CD or positive CD serology

\begin{tabular}{|c|c|c|c|c|c|c|c|c|}
\hline No. & Age (months) & Sex & tTG $\lg A(U / m l)$ & EMA & $A G A \lg A(U / m l)$ & AGA IgG (U/ml) & Risk factor and clinical presentation & $\mathrm{CD}$ diagnosis \\
\hline 1 & 27 & $\mathrm{~F}$ & $>200.0$ & + & 15.0 & 127.0 & Inappetence & $\mathrm{MDB}+$ \\
\hline 2 & 67 & M & 40.7 & + & 8.0 & 18.9 & None & $\mathrm{MDB}+$ \\
\hline 3 & 41 & M & 35.0 & + & 9.3 & 41.0 & $C D$ in the sister & $\mathrm{MDB}+$ \\
\hline 4 & 44 & M & 102.0 & + & 1.4 & 1.1 & None & $\mathrm{MDB}+$ \\
\hline 5 & 24 & $\mathrm{~F}$ & 11.6 & - & 0.2 & 6.4 & Diarrhea & $\mathrm{MDB}+$ \\
\hline 6 & 60 & M & $>200$ & + & 93.0 & 85.5 & None & $\mathrm{HLA}+$ \\
\hline 7 & 44 & $\mathrm{~F}$ & 3.6 & $+/-$ & 1.2 & 17.0 & Growth failure & $\mathrm{DH}$ \\
\hline 8 & 20 & M & 23.0 & + & 1.4 & 9.0 & None & n.p. \\
\hline 9 & 44 & M & 15.0 & + & 1.6 & 9.6 & None & n.p. \\
\hline $10^{\mathrm{a}}$ & 58 & $\mathrm{~F}$ & n.a. & n.a. & п.а. & n.a. & Growth failure Dental enamel defects & $\mathrm{MDB}+, \mathrm{HLA}+$ \\
\hline
\end{tabular}

$M$ male, $F$ female, $t T G \lg A$ anti-transglutaminase antibodies, EMA anti-endomysium antibodies, AGA IgA and lgG: anti-gliadin antibodies; cut -off values for tTG IgA, AGA IgA and AGA IgG: positive: > 10U/ml; borderline: 7-10 U/ml, negative: $<7 \mathrm{U} / \mathrm{ml}$; EMA: “+": positive result; "-": negative result; "+/-": doubt result; n.p. not performed, n.a. not available, HLA+ human leukocyte antigen positivity, $M D B+$ multiple duodenal biopsies positivitym, $D H$ Duhring Dermatitis Herpetiformis ${ }^{a} A S D$ patient who had received a CD diagnosis before the hospitalization in our ASD Unit and for which serological data are not available 
Table 2 Comparison between the prevalence of celiac disease (CD) in our sample and in the Italian pediatric population (Mustalahti et al., [12])

\begin{tabular}{lllll}
\hline & Outcome 1\# & Outcome 2\# & Total & Chi-square \\
& Number of subjects & Number of subjects & Number of subjects & statistic value \\
\hline Group 1 Our study & 10 & 372 & 382 & $0.0131^{*}$ \\
Group 2 Mustalahti et al., 2010 [12] & 29 & 2616 & 2645 \\
Total & 39 & 2988 & 3027 \\
\hline
\end{tabular}

*The result is significant at $p<0.05$

1\# Children with celiac disease (CD) defined as "previously diagnosed biopsy-proven CD patients plus screening detected individuals with both anti-tTG and EMA positivity"

2\# Children without CD defined as "previously diagnosed biopsy-proven CD patients plus screening detected individuals with both anti-tTG and EMA positivity"

for CD. Five patients (50\%) had no symptoms or risk factors correlated with $\mathrm{CD}$ at the time of the serological screening.

\section{Discussion}

Our results indicate that an association between ASD and $\mathrm{CD}$ cannot be excluded. The comparison of our findings with those of previous studies is complex given several differences in the study design (prevalence of ASD in CD children or viceversa), in CD serological screening methodologies (only tTG, both EMA and tTG, AGA and/or EMA and/or tTG antibodies positivity), and in the ASD samples (in terms of size, age of patients, severity of ASD symptoms, diagnostic evaluation and diagnostic criteria). The first studies were performed in the 70'ies [35, 36] and they both did not find any significant association between autism and $\mathrm{CD}$. Some more recent studies also rejected the ASD-CD link. In particular, Batista et al. [17] found no significant differences in the prevalence of CD in 147 ASD patients compared to a group of 2034 children and adolescents originating from the same geographical region and from a similar low-income stratum. In the same work, the prevalence of ASD in 211 patients with CD was $0.95 \%$ and therefore not significantly different from the prevalence of $0.9 \%$ found in the general US population. Pavone et al. [18], in a case-control study, found no cases of autism in 120 patients with $\mathrm{CD}$, and no cases of $\mathrm{CD}$ in 11 patients with autism. On the contrary, Barcia et al. [21] studied retrospectively a large population of 150 randomly selected patients with ASD, and found an increased prevalence of intestinal biopsy-confirmed CD in ASD children (3.3\%) compared to the normal population $(0.9 \%)$. This prevalence $(3.3 \%)$ is quite similar to that found in our work (2.62\%). It is to note that, while Barcia and colleagues included only biopsyconfirmed $\mathrm{CD}$ patients, we considered as $\mathrm{CD}$ patients also the two children without a histological confirmation of the diagnosis, but with both TTG and EMA positive antibodies. We made this choice in agreement with some authors [12] who recently argued that "even if the small intestinal biopsy has an indisputable diagnostic role in the clinical setting, this invasive investigation is not an essential requirement for an epidemiological survey". Besides, in several epidemiological studies the antibody positivity has been considered as the only criterion for CD diagnosis [37-39]. In another recent work, Ludvigsson et al. [22] collected data on 26,995 individuals with CD, 12,304 individuals with inflammation, and 3719 individuals with normal mucosa but positive CD serology (AGA IgA/IgG, EMA and tTG), and compared them with 213,208 age- and sex-matched controls. They found that having a prior diagnosis of ASD was not associated with $\mathrm{CD}(\mathrm{OR}=0.93)$ or inflammation $(\mathrm{OR}=1.03)$, but was related with a markedly increased risk of positive $\mathrm{CD}$ serology $(\mathrm{OR}=4.57)$ [22]. Our findings differed from Ludviggson and colleagues [22] as for the possible association of ASD and CD but it is to note that they estimated the prevalence of ASD diagnosis in subjects that had just performed an intestinal biopsy, while we examined the prevalence of $\mathrm{CD}$ diagnosis after a serological screening in a cohort of subjects diagnosed as ASD. Moreover, the definition of positive $\mathrm{CD}$ serology also differed between Ludviggson et al. [22] and the current investigation since they included subjects with positive AGA IgG and IgA antibodies, while we did not take into account the values of these antibodies. In fact, the main purpose of the present study was to define the prevalence of $\mathrm{CD}$, and AGA antibodies are now considered as the less specific markers of CD in the serological screenings [15].

Among the GI symptoms reported by parents of children with ASD and CD in our sample there were diarrhea and inappetence. The former is one of the most frequently reported GI symptoms in ASD children in several studies $[5,40]$ while the latter has been recently detected as one of the most common GI symptoms in a large sample of pre-schoolers with ASD [41]. Besides, half of the ASD children with CD were asymptomatic at the time of the serological screening: this prevalence could be ascribed not only to the presence of true asymptomatic forms of $\mathrm{CD}$ but also to the severe communication difficulties of non-verbal preschool children with ASD, making them unable to express GI and systemic symptoms suggestive of $\mathrm{CD}$ (e.g. recurrent abdominal pain, abdominal distension, chronic fatigue) $[4,42]$. Otherwise, pre-schoolers with ASD could show their GI-related distress in alternative ways through a greater severity of problem behaviors such as irritability [43], anxiety and affective problems [44, 45], or 
externalizing behaviors (oppositional defiant behaviors and tantrums) [46]. These clinical data contributed to highlight the importance of a serological screening for $\mathrm{CD}$ in young children with ASD, even in absence of clear GI or systemic symptoms or other risk factors related to $\mathrm{CD}$.

A possible limitation of the current study was the absence of data about total IgA level on all ASD patients. Consequently, a certain number of children with $\mathrm{CD}$ and anti IgA deficiency could be not detected leading to an underestimation of $\mathrm{CD}$ prevalence in our ASD population. Another possible source of $C D$ prevalence underestimation in our ASD sample could be ascribed to the young age of patients included that does not allow to identify ASD individuals at genetic risk for CD who develop $C D$ only later. Moreover, we were not able to provide ADOS-G [28] scores, since this instrument was originally used for clinical purposes only (in order to confirm the ASD diagnosis), and therefore his scores were not usable for research purposes.

\section{Conclusion}

Our results indicate that children with ASD may be at increased risk of $\mathrm{CD}$. This finding has implications beyond medical status for children with ASD. Specifically, the fact that GI symptoms suggestive of $C D$ are difficult to express in words by non-verbal or minimally-verbal preschoolers with ASD implies a possible manifestation of GI-related distress through behavioral symptoms, including increased anxiety, decreased social responsiveness, temper tantrums, opposition, sleep problems, food selectivity, aggressive and self-injurious acts [4, 41, 47, 48]. In these cases, if a diagnosis of $\mathrm{CD}$ is confirmed, the treatment of ASD patients for CD with a gluten-free diet may not only alleviate their CD-related symptoms, but also have a positive impact on associated behavioral problems. Therefore, the screening for $\mathrm{CD}$ in young patients with ASD could be important for decreasing current and long-term morbidity, as well as increasing overall health and quality of life.

\section{Abbreviations \\ AGA: Anti-gliadin; ASD: Autism spectrum disorders; CD: Celiac disease; EMA: Anti-endomysium; HLA: Human leukocyte antigen; \\ Ig: Immunoglobuline; tTG: Transglutaminase}

\section{Acknowledgements}

Not applicable.

\section{Funding}

This work was partially supported by grant from the IRCCS Stella Maris Foundation (Ricerca Corrente, and the " $5 \times 1000$ " voluntary contributions, Italian Ministry of Health to FM). SC was partially supported by the Italian Ministry of Health and by Tuscany Region with the grant 'GR-2010-2317873', and by Bando FAS Salute Sviluppo Toscana (ARIANNA Project). ES was partially supported by the Italian Ministry of Health and by Tuscany Region with the grant 'GR-2011-02348280'.

\section{Availability of data and materials}

Dr. Calderoni and Dr. Santocchi had full access to all of the data in the study and takes responsibility for the integrity of the data and the accuracy of the data analysis.

\section{Authors' contributions}

SC and ES participated in the design of the work and wrote the manuscript. LC and AP performed serological tests. TDB wrote the first draft of the manuscript. TDB, FF, MP, AC, RT, collected the data. AN analyzed the data and participated in the design of the work. FM participated in the design of the work, helped to evaluate, edit the manuscript and performed critical revision. All authors read and approved the final manuscript.

\section{Competing interests}

The authors declare that they have no competing interests.

\section{Consent for publication}

All patients or their parents signed an informed consent for publication and agreed for their medical data to be published anonymously.

\section{Ethics approval and consent to participate}

This study was approved by the Research Ethics Committee of the IRCCS Stella Maris Foundation (Pisa, Italy), and performed in compliance with the Declaration of Helsinki and its later amendments. The committee's reference number is not applicable. All patients or their parents signed an informed consent prior to the assessment.

\section{Author details}

${ }^{1}$ IRCCS Stella Maris Foundation, Viale del Tirreno 331, Pisa 56018,

Calambrone, Italy. ${ }^{2}$ Stella Maris Mediterraneo Foundation, Chiaromonte, (PZ), Italy. ${ }^{3} \mathrm{ODFlab}$, Department of Psychology and Cognitive Science, University of Trento, Via Matteo del Ben, 5/B, 38068 Rovereto (TN), Italy. ${ }^{4}$ Department of Translational Research and New Technologies in Medicine and Surgery, University of Pisa, Via Savi 10, 56126 Pisa, Italy. ${ }^{5}$ Department of Clinical and Experimental Medicine, University of Pisa, Pisa, Italy.

Received: 20 July 2016 Accepted: 12 November 2016

Published online: 16 November 2016

\section{References}

1. American Psychiatric Association. Diagnostic and statistical manual of mental disorders. 5th ed. Washington: American Psychiatric Publishing; 2013.

2. Lundström S, Reichenberg A, Melke J, Råstam M, Kerekes N, Lichtenstein P, Gillberg C, Anckarsäter H. Autism Spectrum Disorders and coexisting disorders in a nationwide Swedish twin study. J Child Psychol Psychiatry. 2015;56:702-10.

3. Doshi-Velez F, Ge Y, Kohane I. Comorbidity clusters in autism spectrum disorders: an electronic health record time-series analysis. Pediatrics. 2014;133:e54-63.

4. Buie T, Campbell DB, Fuchs 3rd GJ, Furuta GT, Levy J, Vandewater J, Whitaker AH, Atkins D, Bauman ML, Beaudet AL, Carr EG, Gershon MD, Hyman SL, Jirapinyo P, Jyonouchi H, Kooros K, Kushak R, Levitt P, Levy SE, Lewis JD, Murray KF, Natowicz MR, Sabra A, Wershil BK, Weston SC, Zeltzer L, Winter H. Evaluation, diagnosis, and treatment of gastrointestinal disorders in individuals with ASDs: a consensus report. Pediatrics. 2010;125 Suppl 1:S1-18.

5. McElhanon BO, McCracken C, Karpen S, Sharp WG. Gastrointestinal symptoms in autism spectrum disorder: a meta-analysis. Pediatrics. 2014;133:872-83.

6. Onore C, Careaga M, Ashwood P. The role of immune dysfunction in the pathophysiology of autism. Brain Behav Immun. 2012;26:383-92.

7. Atladóttir HÓ, Pedersen MG, Thorsen P, Mortensen PB, Deleuran B, Eaton WW, Parner ET. Association of family history of autoimmune diseases and autism spectrum disorders. Pediatrics. 2009;124:687-94.

8. Croen LA, Grether JK, Yoshida CK, Odouli R, Van de Water J. Maternal Autoimmune diseases, asthma and allergies, and childhood autism spectrum disorders: a case-control study. Arch Pediatr Adolesc Med. 2005;159:151-7.

9. Keil A, Daniels JL, Forssen U, Hultman C, Cnattingius S, Söderberg KC, Feychting M, Sparen P. Parental autoimmune diseases associated with autism spectrum disorders in offspring. Epidemiology. 2010;21:805-8.

10. Ludvigsson JF, Leffler DA, Bai JC, Biagi F, Fasano A, Green PH, Hadjivassiliou M, Kaukinen K, Kelly CP, Leonard JN, Lundin KE, Murray JA, Sanders DS, Walker MM, Zingone F, Ciacci C. The Oslo definitions for coeliac disease and related terms. Gut. 2013;62:43-52. 
11. Catassi C, Gatti S, Fasano A. The new epidemiology of celiac disease. J Pediatr Gastroenterol Nutr. 2014;59 Suppl 1:S7-9.

12. Mustalahti K, Catassi C, Reunanen A, Fabiani E, Heier M, McMillan S, Murray L, Metzger MH, Gasparin M, Bravi E, Mäki M, Coeliac EU Cluster, Project Epidemiology. The prevalence of celiac disease in Europe: results of a centralized, international mass screening project. Ann Med. 2010;42:587-95.

13. Nenna R, Tiberti C, Petrarca L, Lucantoni F, Mennini M, Luparia RPL, Panimolle F, Mastrogiorgio G, Pietropaoli N, Magliocca FM, Bonamico M. The celiac iceberg: characterization of the disease in primary schoolchildren. J Pediatr Gastroenterol Nutr. 2013;56:416-21.

14. Green PHR, Cellier C. Celiac disease. N Engl J Med. 2007;357:1731-43.

15. Tonutti E, Bizzaro N. Diagnosis and classification of celiac disease and gluten sensitivity. Autoimmun Rev. 2014;13:472-6.

16. Ludvigsson JF, Montgomery SM, Ekbom A, Brandt L, Granath F. Small-intestinal histopathology and mortality risk in celiac disease. JAMA. 2009;302:1171-8.

17. Batista IC, Gandolfi L, Nobrega YKM, Almeida RC, Almeida LM, Campos Junior D, Pratesi R. Autism spectrum disorder and celiac disease: no evidence for a link. Arq Neuropsiquiatr. 2012;70:28-33.

18. Pavone L, Fiumara A, Bottaro G, Mazzone D, Coleman M. Autism and celiac disease: failure to validate the hypothesis that a link might exist. Biol Psychiatry. 1997:42:72-5.

19. Buie T. The relationship of autism and gluten. Clin Ther. 2013;35:57-83.

20. Erickson CA, Stigler KA, Corkins MR, Posey DJ, Fitzgerald JF, McDougle CJ. Gastrointestinal factors in autistic disorder: a critical review. J Autism Dev Disord. 2005:35:713-27.

21. Barcia G, Posar A, Santucci M, Parmeggiani A. Autism and coeliac disease. J Autism Dev Disord. 2007:38:407-8.

22. Ludvigsson JF, Reichenberg A, Hultman CM, Murray JA. A nationwide study of the association between celiac disease and the risk of autistic spectrum disorders. JAMA Psychiatry. 2013;70:1224-30.

23. Reichelt KL, Hole K, Hamberger A, Saelid G, Edminson PD, Braestrup CB, Lingjaerde $\mathrm{O}$, Ledaal $\mathrm{P}$, Orbeck $\mathrm{H}$. Biologically active peptide-containing fractions in schizophrenia and childhood autism. Adv Biochem Psychopharmacol. 1981:28:627-43.

24. Vojdani A, O'Bryan T, Green JA, Mccandless J, Woeller KN, Vojdani E, Nourian AA, Cooper EL. Immune response to dietary proteins, gliadin and cerebellar peptides in children with autism. Nutr Neurosci. 2004;7:151-61.

25. Dosman C, Adams D, Wudel B, Vogels L, Turner J, Vohra S. Complementary, holistic, and integrative medicine: autism spectrum disorder and glutenand casein-free diet. Pediatr Rev. 2013:34:e36-41.

26. Mulloy A, Lang R, O'Reilly M, Sigafoos J, Lancioni G, Rispoli M. Gluten-free and casein-free diets in the treatment of autism spectrum disorders: a systematic review. Res Autism Spectr Disord. 2010;4:328-39.

27. American Psychiatric Association. Diagnostic and statistical manual of mental disorders. 4th ed. Text Revision (DSM-IV-TR). 2000.

28. Lord C, Risi S, Lambrecht L, Cook E, Leventhal BL, DiLavore PC, Pickles A, Rutter M. The autism diagnostic observation schedule-generic: a standard measure of social and communication deficits associated with the spectrum of autism. J Autism Dev Disord. 2000;30:205-23.

29. Husby S, Koletzko S, Korponay-Szabo IR, Mearin ML, Phillips A, Shamir R, Troncone R, Giersiepen K, Branski D, Catassi C, Lelgeman M, Mäki M, RibesKoninckx C, Ventura A, Zimmer KP, ESPGHAN Working Group on Coeliac Disease Diagnosis; ESPGHAN Gastroenterology Committee; European Society for Pediatric Gastroenterology, Hepatology, and Nutrition. European society for paediatric gastroenterology, hepatology and nutrition guidelines for the diagnosis of celiac disease. J Pediatr Gastroenterol Nutr. 2012;54:136-60.

30. Lewis NR, Scott BB. Systematic review: the use of serology to exclude or diagnose coeliac disease (a comparison of the endomysial and tissue transglutaminase antibody tests). Aliment Pharmacol Ther. 2006;24:47-54.

31. Hopper AD, Hadjivassiliou M, Hurlstone DP, Lobo AJ, McAlindon ME, Egner W, Wild G, Sanders DS. What is the role of serologic testing in celiac disease? A prospective, biopsy-confirmed study with economic analysis. Clin Gastroenterol Hepatol. 2008;6:314-20.

32. Hill ID, Dirks MH, Liptak GS, Colletti RB, Fasano A, Guandalini S, Hoffenberg EJ, Horvath K, Murray JA, Pivor M, Seidman EG, North American Society for Pediatric Gastroenterology, Hepatology and Nutrition. Guidelines for the diagnosis and treatment of celiac disease in children: recommendations of the North American society for pediatric gastroenterology, hepatology and nutrition. J Pediatr Gastroenterol Nutr. 2005;40:1-19.

33. Caja S, Mäki M, Kaukinen K, Lindfors K. Antibodies in celiac disease: implications beyond diagnostics. Cell Mol Immunol. 2011:8:103-9.
34. Mendes FB, Hissa-Elian A, Abreu MA, Gonçalves VS. Review: dermatitis herpetiformis. An Bras Dermatol. 2013;88:594-9.

35. Walker-Smith J. Gastrointestinal disease and autism-the results of a survey Paper presented at: Symposium on Autism. Sydney; 1973.

36. McCarthy DM, Coleman M. Response of intestinal mucosa to gluten challenge in autistic subjects. Lancet. 1979;2:877-8.

37. West J, Logan RF, Hill PG, Lloyd A, Lewis S, Hubbard R, Reader R, Holmes GK, Khaw KT. Seroprevalence, correlates, and characteristics of undetected coeliac disease in England. Gut. 2003:52:960-5.

38. Fasano A, Berti I, Gerarduzzi T, Not T, Colletti RB, Drago S, Elitsur Y, Green PH, Guandalini S, Hill ID, Pietzak M, Ventura A, Thorpe M, Kryszak D, Fornaroli F, Wasserman SS, Murray JA, Horvath K. Prevalence of celiac disease in at-risk and not-at-risk groups in the United States: a large multicenter study. Arch Intern Med. 2003;163:286-92.

39. Bingley PJ, Williams AJ, Norcross AJ, Unsworth DJ, Lock RJ, Ness AR, Jones RW, Avon Longitudinal Study of Parents and Children Study Team. Undiagnosed coeliac disease at age seven: population based prospective birth cohort study. BMJ. 2004;328:322-3.

40. Chaidez V, Hansen RL, Hertz-Picciotto I. Gastrointestinal problems in children with autism, developmental delays or typical development. J Autism Dev Disord. 2014;44:1117-27.

41. Fulceri F, Morelli M, Santocchi E, Cena H, Del Bianco T, Narzisi A, Calderoni S, Muratori F. Gastrointestinal symptoms and behavioral problems in preschoolers with Autism Spectrum Disorder. Dig Liver Dis. 2016;48:248-54.

42. Carr EG, Owen-Deschryver JS. Physical illness, pain, and problem behavior in minimally verbal people with developmental disabilities. J Autism Dev Disord. 2007;37:413-24.

43. Bresnahan M, Hornig M, Schultz AF, Gunnes N, Hirtz D, Lie KK, Magnus P, Reichborn-Kjennerud T, Roth C, Schjølberg S, Stoltenberg C, Surén P, Susser E, Lipkin WI. Association of maternal report of infant and toddler gastrointestinal symptoms with autism: evidence from a prospective birth cohort. JAMA Psychiatry. 2015;72:466-74.

44. Maffini V, Fornaroli F, Vincenzi F, Guatelli F, Bizzarri B, Rizzuti L, de'Angelis GL. Autism and gastrointestinal disorders: a close relationship? Dig Liver Dis. 2008;40:A93.

45. Valicenti-McDermott M, McVicar K, Rapin I, Wershil BK, Cohen H, Shinnar S. Frequency of gastrointestinal symptoms in children with autistic spectrum disorders and association with family history of autoimmune disease. J Dev Behav Pediatr. 2006;27:S128-36.

46. Horvath K, Perman JA. Autism and gastrointestinal symptoms. Curr Gastroenterol Rep. 2002;4:251-8.

47. Maenner MJ, Arneson CL, Levy SE, Kirby RS, Nicholas JS, Durkin MS. Brief report: association between behavioral features and gastrointestinal problems among children with autism spectrum disorder. J Autism Dev Disord. 2012;42:1520-5.

48. Mazurek MO, Vasa RA, Kalb LG, Kanne SM, Rosenberg D, Keefer A, Murray DS, Freedman B, Lowery LA. Anxiety, sensory over-responsivity, and gastrointestinal problems in children with autism spectrum disorders. J Abnorm Child Psychol. 2013;41:165-76.

\section{Submit your next manuscript to BioMed Central and we will help you at every step:}

- We accept pre-submission inquiries

- Our selector tool helps you to find the most relevant journal

- We provide round the clock customer support

- Convenient online submission

- Thorough peer review

- Inclusion in PubMed and all major indexing services

- Maximum visibility for your research

Submit your manuscript at www.biomedcentral.com/submit 